IAU Symposium 220: Dark Matter in Galaxies

ASP Conference Series, Vol. **VOLUME***, 2004

S. Ryder, D.J. Pisano, M. Walker, K. Freeman

\title{
X-Ray Constraints on Dark Matter in Galaxy Clusters and Elliptical Galaxies: A View from Chandra and XMM
}

\author{
David A. Buote \\ University of California, Irvine, 4129 Frederick Reines Hall, Irvine, \\ California,92612-4575 U.S.A., buote@uci.edu
}

\begin{abstract}
I review constraints on the radial density profiles and ellipticities of the dark matter obtained from recent X-ray observations with Chandra and XMM of elliptical galaxies and galaxy clusters and discuss their implications, especially for the self-interacting dark matter model.
\end{abstract}

\section{Introduction}

For many years X-ray astronomers have promised to obtain accurate constraints on dark matter in clusters of galaxies and elliptical galaxies. But because of the frustrating limitations of previous X-ray telescopes, only for a very few objects - notably M87 - have precise measurements been possible. It is really a great pleasure to give this review because the promises made many years ago are finally being realized in this wonderful era of X-ray astronomy, where the Chandra and XMM observatories are operating so successfully.

Chandra and XMM have provided for the first time high quality, spatially resolved spectra of the diffuse hot gas of galaxies and clusters because their CCDs combine moderate resolution spectra with very much improved spatial resolution and sensitivity. Chandra provides a more significant jump in spatial resolution while XMM provides a more substantial boost in sensitivity. As a result of these improved capabilities, accurate measurements of the gas temperature as a function of radius exist for many clusters. These measurements provide very interesting constraints on the DM.

Because most of the published results on X-ray studies of dark matter (DM) using Chandra and XMM exist for clusters, in this review I will emphasize the results obtained on the radial DM distributions in clusters. My discussion will be divided up into segments that address the mass distributions inside and outside of cluster cores. I devote the remaining space to elliptical galaxies, particularly NGC 720, where I will discuss X-ray constraints on the ellipticity of DM halos.

\section{Galaxy Clusters}

In galaxy clusters the dominant baryonic component is that of the hot gas, yet it contributes only 10-30 percent to the total mass. Clusters are therefore "DMdominated" and are excellent sites to study the properties of the DM. In fact, in the context of the CDM model, simulations by Dubinski (1998) suggest that clusters are DM-dominated down to less than $1 \%$ of the virial radius $\left(0.01 r_{\text {vir }}\right)$, 
making them especially attractive for studies of the cores of DM halos. Another advantage of studying clusters is that there are a variety of methods that can be used to probe their mass distributions - stellar/galaxy dynamics, gravitational lensing, and dynamics of the hot (X-ray) gas. Each of these methods has certain advantages and disadvantages. The X-ray method, which is the focus here, is primarily hampered by the assumption of hydrostatic equilibrium and questions about the thermodynamic state of the hot gas.

As for the assumption of hydrostatic equilibrium, provided one selects clusters with regular morphologies, hydrodynamic simulations show that the X-ray method is robust, even if the cluster is not in perfect equilibrium (Tsai, Katz, and Bertschinger 1994; Evrard, Metzler, and Navarro 1996; Mathiesen, Evrard, and Mohr 1999). Further support for hydrostatic equilibrium is indicated by the generally good agreement between cluster masses obtained from weak lensing and X-rays (Allen, Schmidt, and Fabian 2002b), though some disagreements with strong lensing remain (Machacek et al. 2002; Xue and Wu 2002).

Regarding the state of the hot gas, mass determinations using X-ray data usually assume the gas is single-phase. Indeed, the Chandra and XMM observations of clusters have justified that assumption (e.g., Peterson et al. 2001; Tamura et al. 2001). These observations have shown that outside cluster cores the hot gas is single-phase. However, within cluster cores the data are consistent with, but do not necessarily require, a limited multiphase medium with a temperature range much less than that expected from a standard multiphase cooling flow. In a few of the cooler systems there is clear evidence for multiphase gas in their cores (M87, Centaurus, NGC 5044). Although the single-phase assumption certainly appears valid outside cluster cores, the situation is less clear within the cores of cool clusters and groups.

For a single-phase gas in hydrostatic equilibrium the calculation of the radial mass distribution from X-ray data is fairly straightforward. Typically, one assumes spherical symmetry and divides up the X-ray image into a series of concentric, circular annuli. Then coronal plasma models are fitted to the annular spectra to infer the temperature and density of the gas as a function of radius. Often this procedure is done by first deprojecting the data using an "onion peeling" method pioneered by Andy Fabian and collaborators. Then parameterized functions are fitted to the radial profiles of the gas density and temperature to allow analytical calculation of the derivatives in the hydrostatic equation. The effects of rotation and magnetic fields are usually ignored but should be negligible (Mathews and Brighenti 2003). Data from next-generation X-ray satellites should provide the first interesting constraints on gas rotation.

\subsection{Outside the Core}

Let us consider first the results obtained with Chandra and XMM for the mass profiles of clusters outside their cores. Perhaps the most impressive example is that of A1413 studied by Pratt and Arnaud (2002). This bright cluster has a very regular X-ray image, the isophotes are moderately flattened, and the radial surface brightness profile shows no structure other than a central enhancement over a single $\beta$ model. These authors obtained an excellent determination of the temperature profile between $100 \mathrm{kpc}$ and $1.5 \mathrm{Mpc}$ (see Figure 1). Pratt \& Arnaud determined the mass profile by examining different parameterizations 

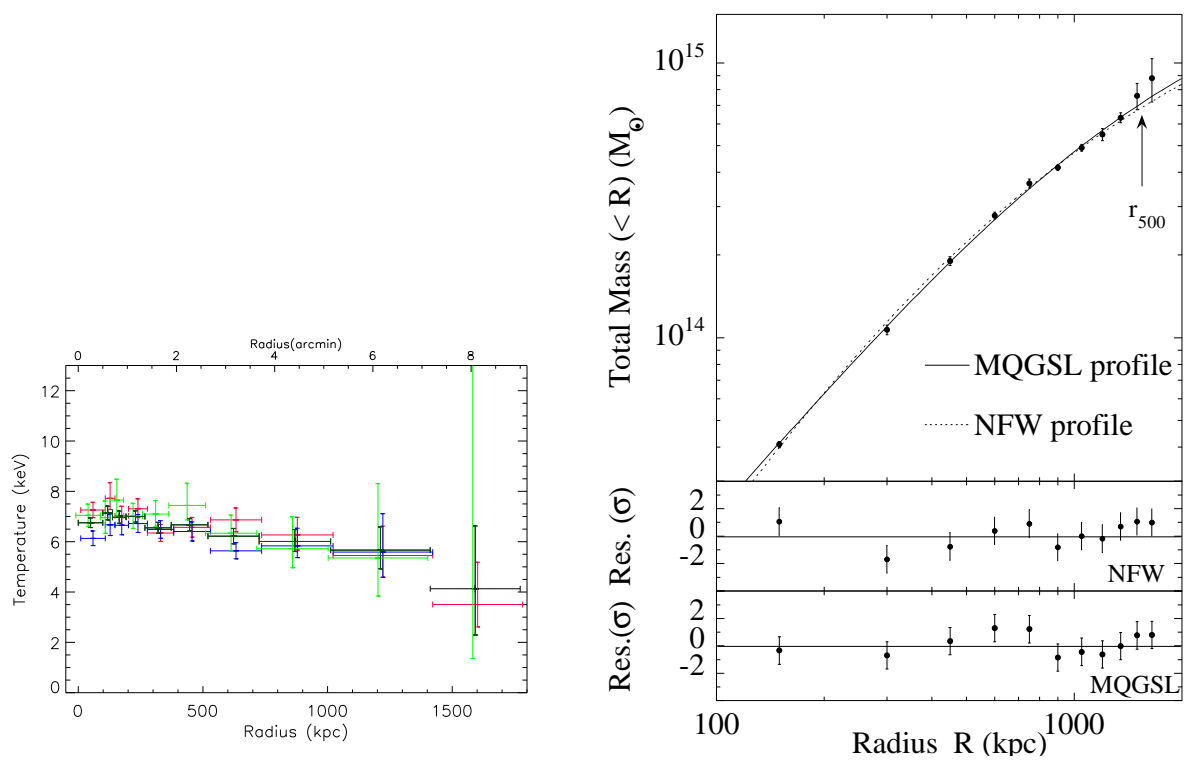

Figure 1. From Pratt \& Arnaud's (2002) analysis of the XMM data of the bright galaxy cluster A1413. (Left Panel) Radial temperature profile of the hot gas, and (Right Panel) the radial mass profile.

for the temperature and density of the hot gas. Outside their central data point, they found the gravitating mass profile is very precisely constrained and is quite insensitive for the specific parameterizations of the gas density and temperature. They find that the NFW and Moore et al. profiles provide good fits to the shape of the gravitating mass profile from $\approx 0.1 r_{\text {vir }}$ out to $0.7 r_{\text {vir }}$ and give reasonable values for the concentration parameter, $c=5.4 \pm 0.2$ (for NFW).

A1835 is another example of a generally relaxed cluster. Schmidt, Allen, and Fabian (2001) obtained good constraints on the mass profile using Chandra data. Their approach to constrain the mass is a slight variation on the procedure mentioned above. They start with an assumed mass profile and use the wellconstrained gas density profile to predict the radial gas temperature profile which is then fitted to the data. Schmidt et al. find acceptable fits to their temperature profile out to $1 \mathrm{Mpc}\left(\approx 0.4 r_{\text {vir }}\right)$ using an NFW model and obtain a reasonable value for the concentration $(c=4.0 \pm 0.6)$. They also find that their mass profile agrees with that obtained from a strong lensing analysis.

Chandra results for A2390 (Figure 2) - another lensing cluster - have been presented by Allen, Ettori, and Fabian (2001). This cluster is not as regular as those just discussed. Instead, there is some substructure in the core and irregularities in the X-ray image on $\approx 100 \mathrm{kpc}$ scales. The radial surface brightness profile even shows a curious break near a radius of $0.5 \mathrm{Mpc}$. Hence, this is certainly a cluster where the assumption of hydrostatic equilibrium is suspect. However, Allen et al. find that the NFW model is a good fit to the data and gives a reasonable value for the concentration $(c=3.3 \pm 1.7)$. Very good agreement is also observed between the X-ray-determined mass and that obtained from strong and weak lensing studies. Thus, whatever departures from hydrostatic 

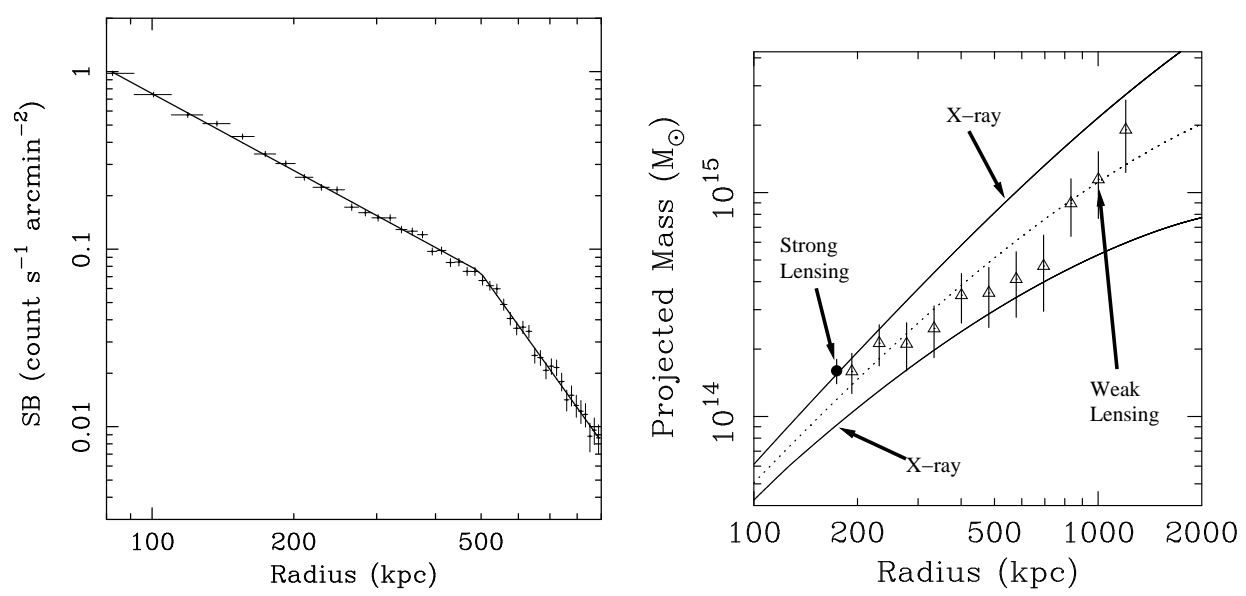

Figure 2. From Allen, Ettori, \& Fabian's (2001) analysis of the Chandra data of the lensing galaxy cluster A2390. (Left Panel) Radial surface profile of the hot gas showing a peculiar break, and (Right Panel) the radial mass profile showing the good agreement between the mass determined by both strong and weak lensing and X-rays.

equilibrium exist in this cluster do not appear to translate to large errors in the derived mass profile.

In addition to the "hot" clusters $(T>5 \mathrm{keV})$ just described, interesting constraints on the mass profiles have also been constrained for cooler clusters. The $X M M$ data of the cool $(T \approx 2 \mathrm{keV})$ cluster A1983 have been analyzed by Pratt and Arnaud (2003). The X-ray image and radial surface brightness profiles are quite regular. The temperature profile of the hot gas is well constrained (and slightly rising) out to $0.5 \mathrm{Mpc}$. Pratt \& Arnaud do find that the NFW profile is a good fit to the data, but the inferred concentration parameter $(c=3.8 \pm 0.7)$ is lower than expected $(\approx 9)$ for this low-mass cluster. They argue that departures from hydrostatic equilibrium are likely the cause of the discrepancy, however, the regularity of the X-ray image does not provide obvious support for that possibility. The authors also find marginal evidence for multi-temperature gas in the central region which should also be considered a possible origin for the anomalous concentration value.

Many more clusters also have been analyzed with Chandra and XMM observations; e.g., A2199 (Johnstone et al. 2002), 3C295 (Allen et al. 2001), and MS1008.1-1224 (Ettori and Lombardi 2003). Overall, NFW (and Moore et al.) profiles are found to be good fits to the mass profiles outside cluster cores $\left(0.3-0.7 r_{\text {vir }}\right)$ obtained from Chandra and XMM observations. Exceptions (particularly A1983) are probably the result of departures from hydrostatic equilibrium and/or multi-temperature gas.

\subsection{Inside the Core}

CDM predicts that DM halos should have approximately a power-law form at small radius $\left(\rho(r) \propto r^{-\alpha}\right)$, though the precise value of the power-law exponent remains controversial. For CDM, simulations indicate that $\alpha$ is between 1 (NFW) and 1.5 (Moore et al.). But observations of LSB galaxies indicate $\alpha \approx 0.5$ in disagreement with CDM. This discrepancy is the motivation of the "self- 

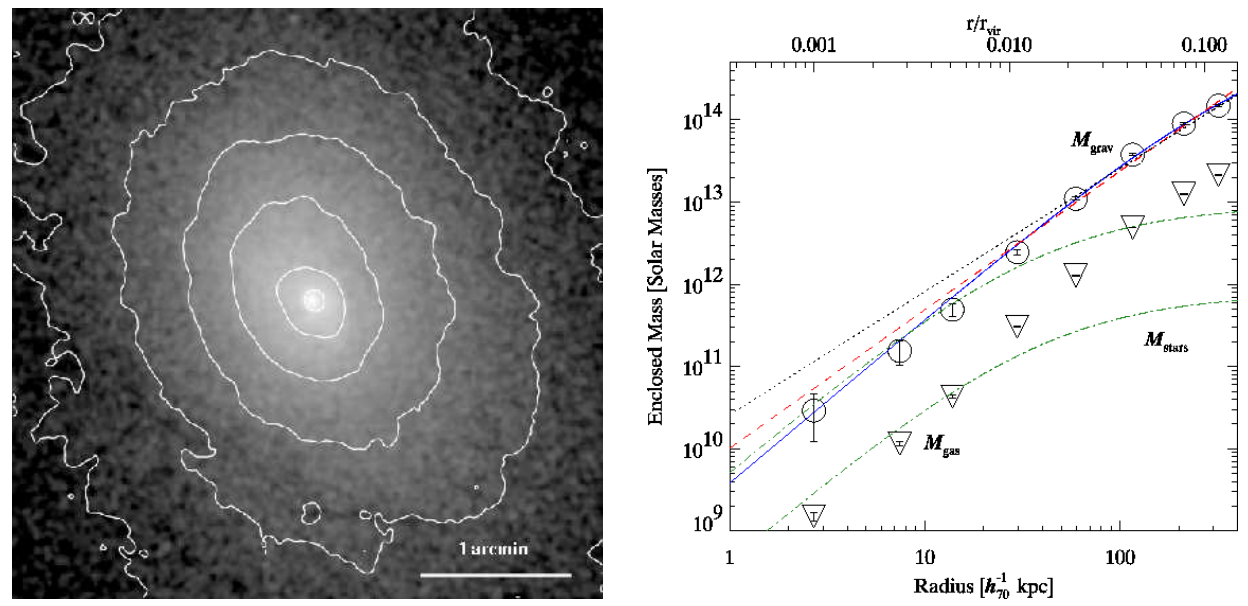

Figure 3. From Lewis et al. (2002,2003): (Left Panel) Chandra ACIS-S image of Abell 2029. The image is $4^{\prime}\left(348 h_{70}^{-1} \mathrm{kpc}\right)$ on a side and has been smoothed with a Gaussian of $\sigma=2^{\prime \prime}$. (Right Panel) Total gravitating mass (data points enclosed with open circles), overlaid with three different mass models: NFW (solid curve), power-law (dashed line), and Moore et al. (dotted curve). The gas mass is plotted as data points enclosed with open triangles. Stellar mass (dot-dashed curves): lower curve assumes a $M_{*} / L_{V}$ of 1 , the upper curve 12 . Note: $r_{\text {vir }}=2.71 h_{70}^{-1} \mathrm{Mpc}$.

interacting" DM model (SIDM) proposed by Spergel and Steinhardt (2000). It is natural to ask whether clusters also have $\alpha \approx 0.5$ since they are DM-dominated deep into their cores. Of course, to address this issue we need to focus on clusters with relaxed cores where the assumption of hydrostatic equilibrium will be valid.

Unfortunately, Chandra observations have shown that nature has provided a very serious roadblock to using X-ray observations to study the mass profiles in cluster cores. Clusters that tend to be the most relaxed systems on hundredkpc and Mpc scales have usually been associated with "cooling flows". But observations with Chandra have shown that in the central regions of cooling flows (i.e., within $r \approx 50 \mathrm{kpc}$ ) the X-ray surface brightness is highly disturbed. The hot gas exhibits filaments and holes - where these holes are often filled by radio emission from an AGN in the central galaxy. Such morphological irregularities clearly call into question the assumption of hydrostatic equilibrium.

Attempts to measure the core mass profile using Chandra observations of clusters with such obvious disturbances have found disagreement with CDM. Although David et al. (2001) obtain $\alpha \approx 1.3$ in the Hydra-A cluster, they obtain a concentration parameter $(c=12.3 \pm 0.2)$ that is higher than expected. In the core of A1795 Ettori et al. (2002) find $\alpha=0.59 \pm 0.15$ in clear disagreement with CDM.

It is difficult to evaluate these results for Hydra-A and A1795 since departures from hydrostatic equilibrium could easily explain the discrepancies with CDM. Clearly, it is essential to find X-ray clusters that are relaxed in their cores. 
Some such clusters have now been identified by considering those systems that are known to be be relaxed on hundred-kpc and Mpc scales but also do not have a strong radio source in the central galaxy.

One such cluster is A2029 (Figure 3) which Chandra shows is indeed regularly shaped all the way down into its core (Lewis, Stocke, and Buote 2002; Lewis, Buote, and Stocke 2003). This massive cluster is also nearby $(z=0.0767)$ and very bright, making it an ideal target for X-ray analysis of its core mass distribution. The Chandra spectra of A2029 extracted within thin annuli from the center out to $r \approx 300 \mathrm{kpc}$ are well-fitted by a single temperature component. This is a key result since one may have expected possible complications from a multiphase gas in the center of a cooling flow.

These authors find that the mass profile of A2029 is reasonably well described by a simple power law that is quite insensitive to different parameterizations of the measured gas density and temperature profiles. The exponent of the power-law fit to the gravitating mass profile is $1.68 \pm 0.02$. This translates to $\alpha=1.32 \pm 0.02$ for the mass density profile, very similar to the NFW/Moore values, yet very significantly larger than the values $\alpha \approx 0.5$ obtained for LSB galaxies. The NFW model is a better fit to the mass profile than either a power-law or the Moore et al. model - though a fit of similar quality is obtained for the Hernquist profile. The inferred concentration parameter is reasonable $(c=5.9 \pm 0.3)$. After subtracting out the contribution to the gravitating mass from the hot gas and stars, it is found that the DM has very similar properties to that of the gravitating matter: $\alpha=1.25 \pm 0.03$ for a power-law mass model and $c=4.6 \pm 0.4$ for NFW. (Note: these results quoted for A2029 include an extra data point at large radius not used by Lewis et al. 2003 and thus differ slightly from the previously published results.)

In Buote and Lewis (2003) we present a Chandra observation of A2589 which is another cluster that is found to be regularly shaped all the way into into its core. Our Chandra observation unfortunately only yielded 8 ks of good data, and thus the constraints are not as precise as those obtained for A2029. Like A2029, the hot gas in A2589 is consistent with a single-phase gas. The gas density and temperature are well constrained and described by simple functions - the gas is consistent with being isothermal. The results for the gravitating mass profile are quite consistent with those obtained for A2029. A power-law fit to the mass profile yields $\alpha=1.37 \pm 0.14$ for the mass density. A better fit is provided by NFW which is a good fit down to $\approx 0.01 r_{\text {vir }}$ with a reasonable concentration parameter $(c=6.6 \pm 2.5)$. Similar results are obtained for the DM: $\alpha=1.35 \pm 0.21$ for a power-law mass model and $c=4.9 \pm 2.4$ for NFW.

Similar agreement with CDM is also observed for some clusters (Allen, Schmidt, and Fabian 2002a; Arabadjis, Bautz, and Garmire 2002) that are not quite so obviously relaxed in their cores as A2029 and A2589 but are clearly not as disturbed as systems like Hydra-A and A1795. Hence, in contrast to the clusters with unrelaxed cores, the core DM profiles inferred for clusters with relaxed cores appear to be quite consistent with CDM. 

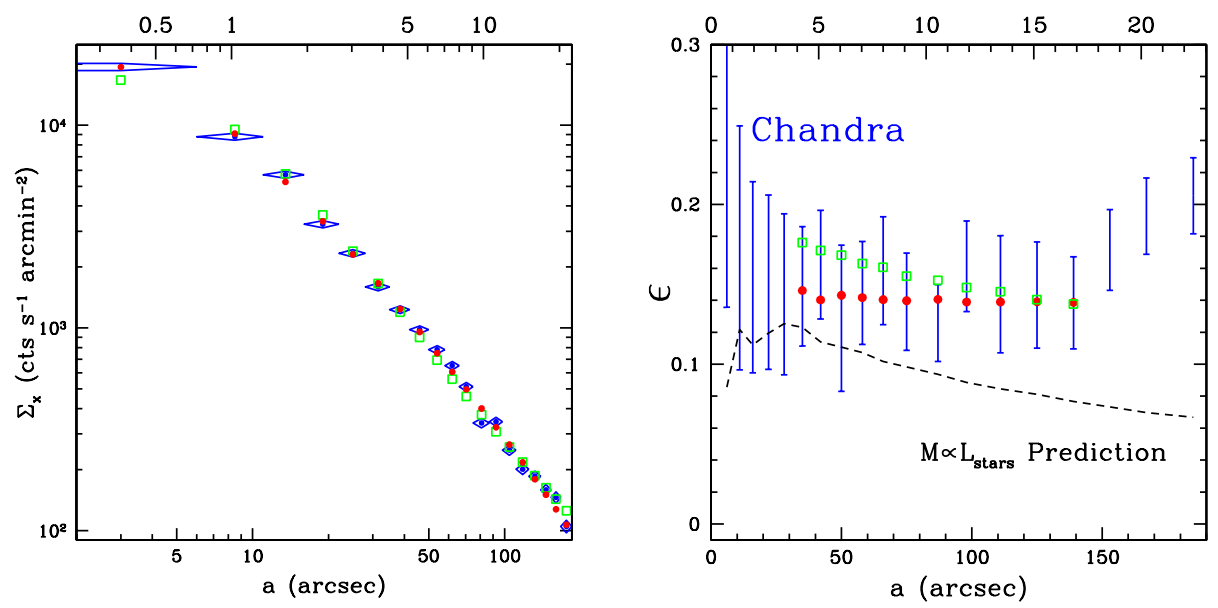

Figure 4. From Buote et al.'s (2002) analysis of the Chandra data of the elliptical galaxy NGC 720. (Left panel) Radial surface brightness profile denoted by diamonds (blue). Also shown are the best-fitting radial profiles generated by (oblate) DM halos corresponding to a $\rho \sim$ $a^{-2}$ profile (filled circles - red) and an NFW profile (open squares green). (Right panel) X-ray ellipticities predicted by these DM models. The error bars (blue) are the values of $\epsilon_{x}$ measured from the source-free chandra data, and the dashed line is the prediction if mass follows the stars. We express $a$ in $\mathrm{kpc}$ on the top axis.

\section{Shapes of DM Halos in Elliptical Galaxies}

Unfortunately, since the X-ray halos of individual elliptical galaxies are much fainter than those of clusters and groups of galaxies, it is much more difficult to obtain interesting constraints on the gravitating mass in elliptical galaxies using X-rays. In fact, only for NGC 720 has a detailed analysis of the mass distribution using Chandra data been published so far.

The ellipticities of DM halos provide important constraints on the nature of the DM in cosmological models essentially independent of $\Omega_{m}$ or $\Lambda$ (Frenk et al. 1988; Bullock 2002). Nearly spherical halos are expected for the cores of DM halos if the dark matter is self-interacting (Davé et al. 2001). Highly flattened (nearly disk-like) DM halos occur in models where the DM is in the form of cold, molecular gas (Pfenniger, Combes, and Martinet 1994). Halos formed in a $\Lambda$ CDM Universe are moderately flattened with typical ellipticities $0.3-0.5$, though they are rounder if there is significant "warm" DM (Bullock 2002).

In contrast to the case of spherical symmetry discussed so far, there is no simple analytic solution of the equation of hydrostatic equilibrium for ellipsoidal mass distributions. However, the added complexity of ellipsoidal symmetry does offer some subtle rewards. For a single-phase gas in hydrostatic equilibrium the volume X-ray emissivity and the gravitational potential have identical shapes independent of the temperature profile (An "X-ray Shape Theorem" - Buote and Canizares 1994; Buote and Canizares 1996). This allows a robust "Geometric Test" of the hypothesis that mass follows the optical light. One just 
deprojects the stellar image and computes the potential assuming mass follows the optical light. The shape of the potential is then compared to the shape of the deprojected X-ray image independent of the gas temperature profile.

This test for DM has been applied to a Chandra observation of the nearby ( $D=25 \mathrm{Mpc}$ ) galaxy NGC 720 (Buote et al. 2002). This is an isolated E4 galaxy that is fairly bright in X-rays. The Chandra image of this galaxy revealed over 40 discrete sources embedded within the diffuse emission within $2.5^{\prime}$ of the galaxy center. These sources, most of which were undetected by previous observations, are wonderful for studying the properties of X-ray binaries, but are a nuisance for studies of DM. These sources were removed from the image and replaced with estimates of the local diffuse emission. The image of NGC 720 cleaned of discrete sources displays X-ray isophotes with moderate flattening and orientations that are slightly offset from the optical.

Starting at the center of the galaxy, the positional angles (PAs) of the isophotes slowly twist $\approx 20^{\circ}$ away from the optical major axis out to $r \approx 15 \mathrm{kpc}$. There is a further twist at larger radii. Here we focus on the region within $\approx 15 \mathrm{kpc}$. If the gas is in hydrostatic equilibrium, since this twist does not occur in the stellar image it implies the existence of a massive DM halo according to the Geometric Test mentioned above.

The X-ray ellipticity profile is consistent with a constant value $(\approx 0.15)$ out to $\approx 15 \mathrm{kpc}$ at which point it increases. Again, we restrict our discussion to the region within this radius. The X-ray ellipticities are much less than the stellar values $(\approx 0.4)$. But they are larger than predicted by the Geometric Test (Figure 4). The mass-follows-optical-light hypothesis is rejected - using ellipticities alone - at slightly more than the $2 \sigma$ level.

The evidence for DM provided by the X-ray PA twist and ellipticities cannot be explained away by MOND. First the need for DM occurs already in the region where Newtonian gravity should dominate. Second, the potential shapes in MOND are the same as in Newtonian theory. Note that gas rotation, which is not accounted for in this analysis, is not expected to be important for NGC 720 (Buote et al. 2002).

We find that the ellipticity of the gravitating mass distribution, which is dominated by the DM, is not very sensitive to the mass model (Figure 4). We find $\epsilon=0.37 \pm 0.03$ for $\rho \sim a^{-2}$ and $\epsilon=0.41 \pm 0.02$ for NFW. Note that if the stars make a sizable contribution to the potential (which is not supported by the Chandra data) then the ellipticity of the DM will be larger than that of the gravitating matter.

The DM ellipticity inferred for NGC 720 is consistent with CDM predictions but is very inconsistent with the round halos predicted by SIDM and the very flat halos predicted if the DM is cold gas. The PA twist, particularly within $\approx 15 \mathrm{kpc}$ strongly suggests the DM is triaxial - the twist appears to agree with the triaxial model of NGC 720 proposed by Romanowsky and Kochanek (1998).

Although these results give nice agreement with CDM predictions, it is essential to verify them using independent methods and with other galaxies. The stellar velocity field could offer such an independent test since the strongly triaxial mass distribution indicated by the X-ray data should produce a highly asymmetric stellar velocity field (T. Statler, private communication). We are 
currently analyzing new observations of the elliptical galaxies NGC 1332 and NGC 3923 which will provide key comparisons to mass properties of NGC 720.

We emphasize that NGC 720 shows the importance of detecting and accounting for discrete sources embedded in the diffuse emission. Statler and McNamara (2002) argue that the Chandra measurements of highly flattened X-ray isophotes of the elliptical galaxy NGC 1700 indicate that rotation must be dynamically important in the hot gas. However, these authors detect only 2-3 discrete sources in their system, and the X-ray ellipticity profile they derive matches the optical ellipticities over almost the whole radius range investigated. This strongly suggests that that the morphology of the X-ray emission of NGC 1700 is strongly biased by the unresolved discrete sources.

\section{Conclusions}

Presently, the key result obtained from Chandra and XMM studies of DM in clusters and ellipticals is that the radial density profiles in galaxy clusters and the ellipticity of the DM halo (for NGC 720) agree well with CDM predictions. In particular, the DM profiles measured deep down into the cores of the relaxed galaxy clusters A2029 and A2589 rule out an important contribution from selfinteracting DM of the kind proposed to account for DM in the cores of LSB galaxies.

Acknowledgments. I thank the conference organizers for the invitation to provide this review and for arranging such an excellent meeting. I also thank my collaborators on X-ray studies of DM for their contributions to some of the papers discussed in this review.

\section{References}

Allen, S. W., Ettori, S., and Fabian, A. C. 2001, MNRAS, 324, 877

Allen, S. W., Schmidt, R. W., and Fabian, A. C. 2002a, MNRAS, 335, 256

Allen, S. W., Schmidt, R. W., and Fabian, A. C. 2002b, MNRAS, 334, L11

Allen, S. W. et al. 2001, MNRAS, 324, 842

Arabadjis, J. S., Bautz, M. W., and Garmire, G. P. 2002, ApJ, 572, 66

Bullock, J. S. 2002, in The shapes of galaxies and their dark halos, Proceedings of the Yale Cosmology Workshop "The Shapes of Galaxies and Their Dark Matter Halos", New Haven, Connecticut, USA, 28-30 May 2001. Edited by Priyamvada Natarajan. Singapore: World Scientific, 2002, ISBN 9810248482, p.109, p. 109

Buote, D. A. and Canizares, C. R. 1994, ApJ, 427, 86

Buote, D. A. and Canizares, C. R. 1996, ApJ, 457, 177

Buote, D. A., Jeltema, T. E., Canizares, C. R., and Garmire, G. P. 2002, ApJ, 577, 183 
Buote, D. A. and Lewis, A. D. 2003, ApJ, submitted

Davé, R., Spergel, D. N., Steinhardt, P. J., and Wandelt, B. D. 2001, ApJ, 547, 574

David, L. P., Nulsen, P. E. J., McNamara, B. R., Forman, W., Jones, C., Ponman, T., Robertson, B., and Wise, M. 2001, ApJ, 557, 546

Dubinski, J. 1998, ApJ, 502, 141

Ettori, S., Fabian, A. C., Allen, S. W., and Johnstone, R. M. 2002, MNRAS, 331,635

Ettori, S. and Lombardi, M. 2003, A\&A, 398, L5

Evrard, A. E., Metzler, C. A., and Navarro, J. F. 1996, ApJ, 469, 494

Frenk, C. S., White, S. D. M., Davis, M., and Efstathiou, G. 1988, ApJ, 327, 507

Johnstone, R. M., Allen, S. W., Fabian, A. C., and Sanders, J. S. 2002, MNRAS, 336,299

Lewis, A. D., Buote, D. A., and Stocke, J. T. 2003, ApJ, 586, 135

Lewis, A. D., Stocke, J. T., and Buote, D. A. 2002, ApJ, 573, L13

Machacek, M. E., Bautz, M. W., Canizares, C., and Garmire, G. P. 2002, ApJ, 567,188

Mathews, W. G. and Brighenti, F. 2003, ARA\&A, 41, 191

Mathiesen, B., Evrard, A. E., and Mohr, J. J. 1999, ApJ, 520, L21

Peterson, J. R. et al. 2001, A\&A, 365, L104

Pfenniger, D., Combes, F., and Martinet, L. 1994, A\&A, 285, 79

Pratt, G. W. and Arnaud, M. 2002, A\&A, 394, 375

Pratt, G. W. and Arnaud, M. 2003, A\&A, 408, 1

Romanowsky, A. J. and Kochanek, C. S. 1998, ApJ, 493, 641

Schmidt, R. W., Allen, S. W., and Fabian, A. C. 2001, MNRAS, 327, 1057

Spergel, D. N. and Steinhardt, P. J. 2000, Physical Review Letters, 84, 3760

Statler, T. S. and McNamara, B. R. 2002, ApJ, 581, 1032

Tamura, T. et al. 2001, A\&A, 365, L87

Tsai, J. C., Katz, N., and Bertschinger, E. 1994, ApJ, 423, 553

Xue, S. and Wu, X. 2002, ApJ, 576, 152 